CERN-EP/98-129

August 7, 1998

\title{
Influence of dissolved gas and temperature on the light yield of new liquid scintillators ${ }^{\star}$
}

\author{
S. Buontempo ${ }^{1}$, F. Galeazzi ${ }^{2}$, S.V. Golovkin ${ }^{3}$, G. Martellotti ${ }^{2}$, \\ A.M. Medvedkov ${ }^{3}$, G. Penso ${ }^{2}$, A.S. Solovjev ${ }^{4}$, and V.G. Vasil'chenko ${ }^{3}$
}

\begin{abstract}
Sixteen new liquid scintillators, emitting green light, were studied. They are based on four solvents combined with four dopants. The influence of different gas atmospheres was studied. In particular it was shown that by keeping these liquid scintillators in vacuum or in a neutral gas, the light yield increases up to $32 \%$ at $20{ }^{\circ} \mathrm{C}$ and for the best solvent-dopant combinations. The dependance of the light yield on temperature was also studied for these scintillators. In the $20-60{ }^{\circ} \mathrm{C}$ interval, some exhibit a light yield variation of $\sim 3 \%$ which is smaller than that of the NE $102 \mathrm{~A}$ plastic scintillator.
\end{abstract}

PACS: 29.40.Mc; 29.40.Gx; 29.40.Vj

Keywords: Liquid scintillators; Scintillation detectors; Tracking and position-sensitive detectors; Calorimeters.

(Submitted to Nuclear Instruments \& Methods A)

* This work is part of the CERN Research and Development programme RD46.

${ }^{1}$ Università "Federico II" and INFN, Napoli, Italy.

${ }^{2}$ Università di Roma "La Sapienza" and INFN, Roma, Italy.

${ }^{3}$ IHEP, Protvino, Russian Federation.

4 JINR, Dubna, Russian Federation. 


\section{Introduction}

During the last few years different tracking detector prototypes [1-8] based on capillaries filled with liquid scintillators (LSs) which emit light in the green region have been developed and successfully tested. These scintillators present a high light output $[2,3,8]$, a large Stokes shift which results in low self-absorbtion, a short decay time of the order of $6-8 \mathrm{~ns}[3,8]$, and a high radiation hardness of the order of $100 \mathrm{Mrad}$ [4]. With these LSs the tracking devices tested provide a hit density along a minimum ionizing track of $\sim 6.5(3) \mathrm{hits} / \mathrm{mm}$, at a distance of $20(150) \mathrm{cm}$ from the readout system.

In the present paper, we show how the light yield of these LSs depends on the temperature and on the presence of dissolved gas.

\section{Experimental method}

In order to measure the light yield of various scintillators in different gas atmospheres and at different temperatures, the set-up shown in Fig. 1 was used. The LSs $\left(\sim 2 \mathrm{~cm}^{3}\right)$ were placed in a cylindrical glass cuvette $(1.6 \mathrm{~cm}$ in diameter), directly on the input window of a FEU-84-3 photomultiplier with a multi-alkaline photocathode. The scintillators were irradiated with a ${ }^{90} \mathrm{Sr} \beta$-source, and the output current of the photomultiplier was measured with a precison of $\pm 5 \%$. The emission spectrum of the scintillators and the spectral sensitivity of the photocathode were previously measured and taken into account in order to deduce the light yield from the measured photomultiplier current. The overall error on the light yields is estimated to be $\pm 10 \%$. To calibrate our system the light yield emitted by an anthracene crystal was measured in the same experimental conditions, and used as a reference value. To check the entire system the light yield of a standard polystyrene scintillator containing $1.5 \%$ of p TP and $0.01 \%$ of POPOP was measured. This measurement was performed with two cylindrical $(1.6 \mathrm{~cm}$ in diameter) scintillators having a thickness of $0.5 \mathrm{~cm}$ and $1 \mathrm{~cm}$, respectively. The measured light yield for the $0.5 \mathrm{~cm}$ thick sample was $\sim 45 \%$ of the anthracene crystal, whilst for the 1 $\mathrm{cm}$ thick sample this was only $\sim 25 \%$, due to the strong self-absorbtion of the POPOP for the short wavelength emission. These results are in good agreement with the known characteristics of this polystyrene scintillator. In Fig. 2 the spectral sensitivity of the photocathode is shown, together with the emission spectra of the anthracene crystal, of the polystyrene scintillator, and of a typical green-emitting liquid scintillator we are considering.

\section{Results}

Different types of LSs obtained by combining four solvents (1MN ${ }^{1}$, IPN, DIPN and $1 \mathrm{PN}$ ) and four dopants (R6 ${ }^{2}, \mathrm{R} 45, \mathrm{R} 39$ and $\left.3 \mathrm{M}-15\right)$ were studied. The concentration of dopants in solvents was $3 \mathrm{~g} / 1$. As regards light yield, the best LSs with a high refractive index [9] were found to be those based on $1 \mathrm{MN}$ and IPN solvents, as shown in Table 1.

\footnotetext{
${ }^{1}$ In the Appendix a list of the abbreviations used for solvents and dopants is given.

${ }^{2}$ R6, R45, R39 and 3M-15 are trade marks of the Geosphaera Research Centre, Vavilova 70/2, Moscow, 117261, Russian Federation.
} 
Table 1: Scintillation properties of the liquid scintillators, at $T=20^{\circ} \mathrm{C} . L_{a}$ and $L_{v}$ are their light yield in air and vacuum, respectively, expressed as a percentage of that of anthracene. $k=L_{v} / L_{a}$. In the last column the derivative of the light yield in air with respect to the temperature at $T=20^{\circ} \mathrm{C}$ is given.

\begin{tabular}{lcccc}
\hline Scintillator & $L_{a}(\%)$ & $L_{v}(\%)$ & $k$ & $d L_{a} / d T\left(\% /{ }^{\circ} \mathrm{C}\right)$ \\
\hline $1 \mathrm{MN}+\mathrm{R} 6$ & 46.8 & 59.2 & 1.27 & +0.024 \\
1MN + R45 & 47.3 & 58.6 & 1.24 & -0.125 \\
1MN + R39 & 47.9 & 63.2 & 1.32 & -0.042 \\
$1 \mathrm{MN}+3 \mathrm{M}-15$ & 44.2 & 54.9 & 1.24 & -0.024 \\
\hline $\mathrm{IPN}+\mathrm{R} 6$ & 46.7 & 61.8 & 1.32 & -0.054 \\
IPN + R45 & 44.6 & 56.6 & 1.27 & -0.149 \\
IPN + R39 & 46.4 & 59.5 & 1.28 & -0.058 \\
IPN + 3M-15 & 49.5 & 60.0 & 1.21 & -0.060 \\
\hline DIPN + R6 & 38.2 & 48.2 & 1.26 & -0.024 \\
DIPN + R45 & 33.7 & 42.5 & 1.26 & -0.038 \\
DIPN + R39 & 35.0 & 43.7 & 1.25 & -0.072 \\
DIPN + 3M-15 & 36.7 & 44.6 & 1.21 & -0.092 \\
\hline 1PN + R6 & 34.8 & 37.4 & 1.07 & -0.028 \\
1PN + R45 & 26.4 & 29.0 & 1.10 & -0.083 \\
1PN + R39 & 31.9 & 35.3 & 1.11 & -0.068 \\
1PN + 3M-15 & 31.0 & 34.7 & 1.12 & -0.030 \\
\hline
\end{tabular}

\subsection{Influence of gas atmospheres on scintillation light output}

With the set-up shown in Fig. 1, the dependance of the scintillation light yield on different gas atmospheres, at $20^{\circ} \mathrm{C}$, was measured for the LS composed of $1 \mathrm{MN}+\mathrm{R} 6$. The light yield was measured as a function of time, during the operations described hereafter, and shown in Fig. 3. The LS was first degassed by pumping for three hours. In this first time interval the light yield increases by a factor of $\sim 1.27$ with respect to light yield in air, at atmospheric pressure. Different gases are then introduced in the set-up at a pressure of $1.1 \mathrm{~atm}$. In less than one hour the light yield reaches a plateau which depends on the kind of gas introduced. Finally, after five hours from the beginning of the pumping, air at atmospheric pressure was again introduced in the setup. During this last phase the light yield drops rapidly and reaches the original value in about 24 hours. The light yield of $1 \mathrm{MN}+\mathrm{R} 6$, measured in the central plateau region (Fig. 3), is shown in Fig. 4 for different gases. For air and Ar the dependance of the light yield of $1 \mathrm{MN}+\mathrm{R} 6$ on the gas pressure was also measured and is shown in Fig. 5 . From this curve it can be seen that for normal variations in atmospheric pressure the light yield of LSs in air can vary by a few per cent. This effect, which could be relevant in an electromagnetic or hadronic calorimeter using this LS, is strongly reduced if the LS is degassed and then kept in a neutral gas atmosphere such as Ar or Ne.

For other combinations of solvents and dopants, only the light yield in air and in vacuum were measured. These results are shown in Table 1. 


\subsection{Influence of temperature on scintillation light output}

For some applications, such as high-energy physics calorimetry using LSs, the temperature stability of the scintillating light yield is an important factor. The dependence of the light yield of $1 \mathrm{MN}-$ IPN- and DIPN-based LSs, in the temperature interval between $-20^{\circ} \mathrm{C}$ to $+80{ }^{\circ} \mathrm{C}$ was measured. This interval was limited at low temperature by LS freezing, and at high temperature by LS evaporation. The results of these measurements are shown in Figs. 6, 7 and 8, for LSs in air and in vacuum. In this temperature interval the light yield of the LSs varies between a few per cent and $30 \%$. In the interval between +20 and $+60{ }^{\circ} \mathrm{C}$ the variation of the light yield for most of the LSs is comparable or even smaller than the corresponding one for the NE $102 \mathrm{~A}$ plastic scintillator which is $\sim 5 \%$ [10]. For example, for $1 \mathrm{MN}+\mathrm{R} 6$ in air the light yield varies in this temperature interval by $\sim 3 \%$.

As a measure of the light yield stability in normal working conditions, the derivative of the light yield with respect to the temperature at $T=20{ }^{\circ} \mathrm{C}$ is calculated. In the last column of Table 1 the values for all possible combinations of solvents and dopants are given.

The scintillation of pure solvents (1MN, IPN, DIPN and 1PN) in air and vacuum was also measured $^{3}$. The results, as a function of the temperature, are shown in Fig. 9. This light yield strongly decreases with increasing temperature, in the interval between -20 and $+80^{\circ} \mathrm{C}$, in contrast with that observed for the LSs.

\section{Conclusions}

Some properties of new LSs, emitting light in the green region were studied. Four solvents (1MN, IPN,DIPN and 1PN) combined with four different dopants (R6, R45, R39 and 3M-15) were considered. The influence of gas atmospheres on light yield was studied. In particular, it was shown that by eliminating the air dissolved in the LSs, the light yield increases up to $32 \%$ at $20^{\circ} \mathrm{C}$ and for the best LSs, which are based on $1 \mathrm{MN}$ and IPN solvents. Under these conditions a light yield of $\sim 60 \%$ of that of anthracene is attained. This enhancement remains practically unchanged if the LSs are kept in a neutral atmosphere such as Ar or Ne. The dependance of the light yield on temperature was also studied for different solvent-dopant combinations. For some particular LSs, such as $1 \mathrm{MN}+\mathrm{R} 6$, in the temperature interval $20-60{ }^{\circ} \mathrm{C}$, a light yield variation of $\sim 3 \%$ was found, which is smaller than that of the NE $102 \mathrm{~A}$ plastic scintillator.

\section{Acknowledgments}

The authors would like to thank G.I. Britvich for his help during the light output calibration.

\footnotetext{
${ }^{3}$ The pure solvents emit light in the blue region. Their emission spectra have not been measured with the same precision as that of LSs. This results in a further error of $\pm 10 \%$ on the light yield of pure solvents, caused by the correction for the spectral sensitivity of the photocathode.
} 


\section{Appendix}

List of abbreviations used for solvents and dopants. The peak emission wavelength $\lambda$ is reported.

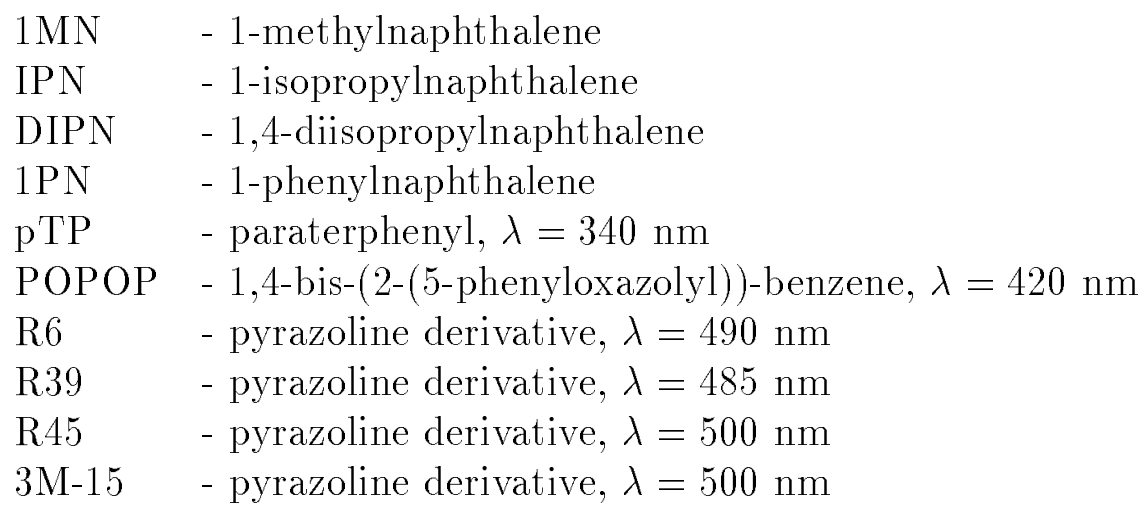

\section{References}

[1] C. Cianfarani et al., Nucl. Instr. and Meth. A339 (1994) 449.

[2] S. Buontempo, et al., Nucl. Instr. and Meth. A360 (1995) 7.

[3] A. Cardini et al., Nucl. Instr. and Meth. A361 (1995) 129.

[4] S.V. Golovkin et al., Nucl. Instr. and Meth. A362 (1995) 283.

[5] P. Annis et al., Nucl. Instr. and Meth. A367 (1995) 377.

[6] F. Ferroni et al., Nucl. Instr. and Meth. A368 (1995) 224.

[7] G. Martellotti et al., Proceedings SPIE, 2551 (1995) 53.

[8] S.V. Golovkin et al., Preprint IHEP 96-13, Protvino 1996.

[9] V.G. Vasil'chenko et al., Instr. and Exper. Tech., 40 (1997) 175.

[10] J.F. Cameron et al., Nucl. Electron., IAEA, 1 (1962) 95. 


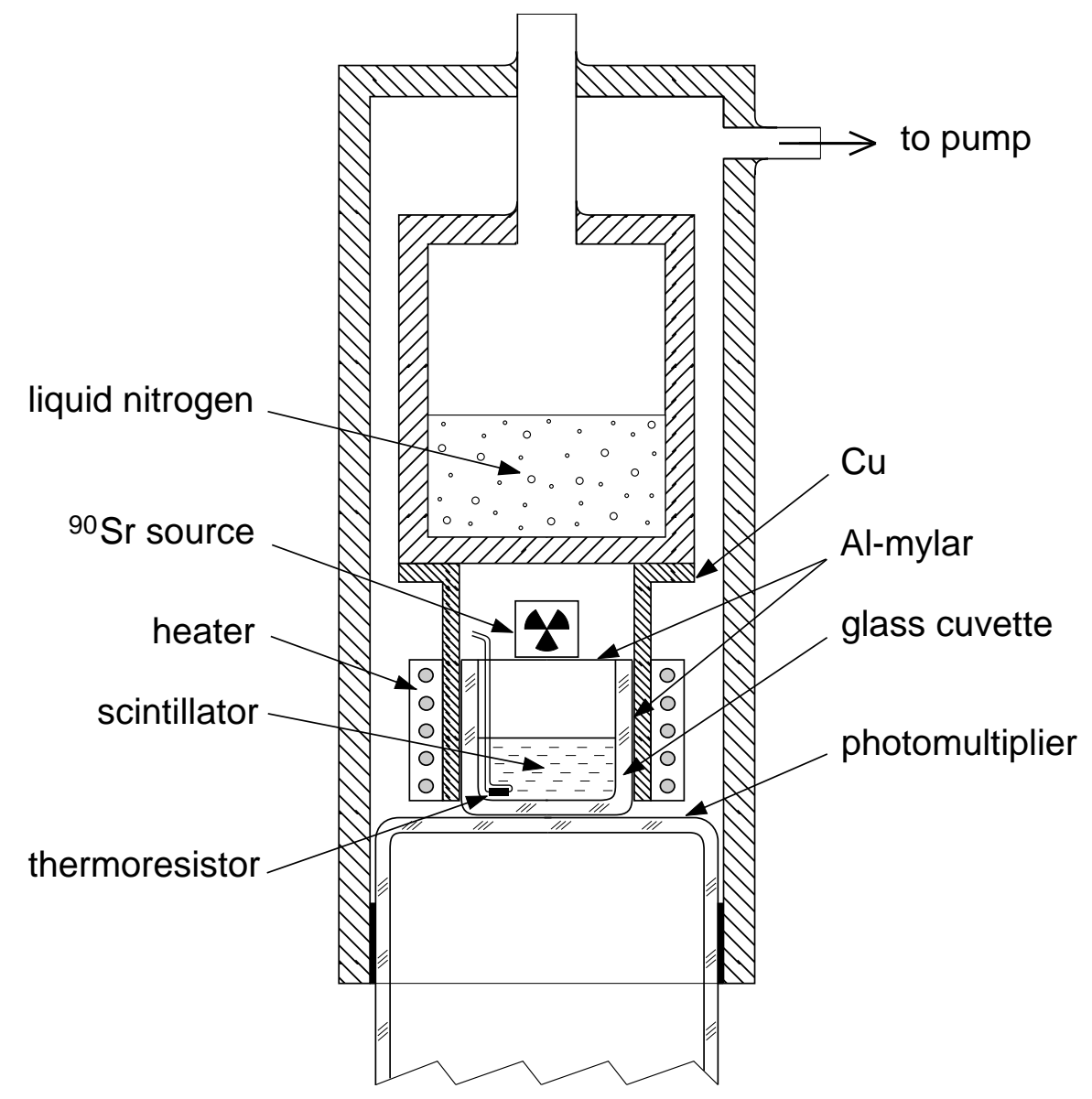

Figure 1: Set-up used to measure the light yield of the liquid scintillators in the different conditions reported in the text. 


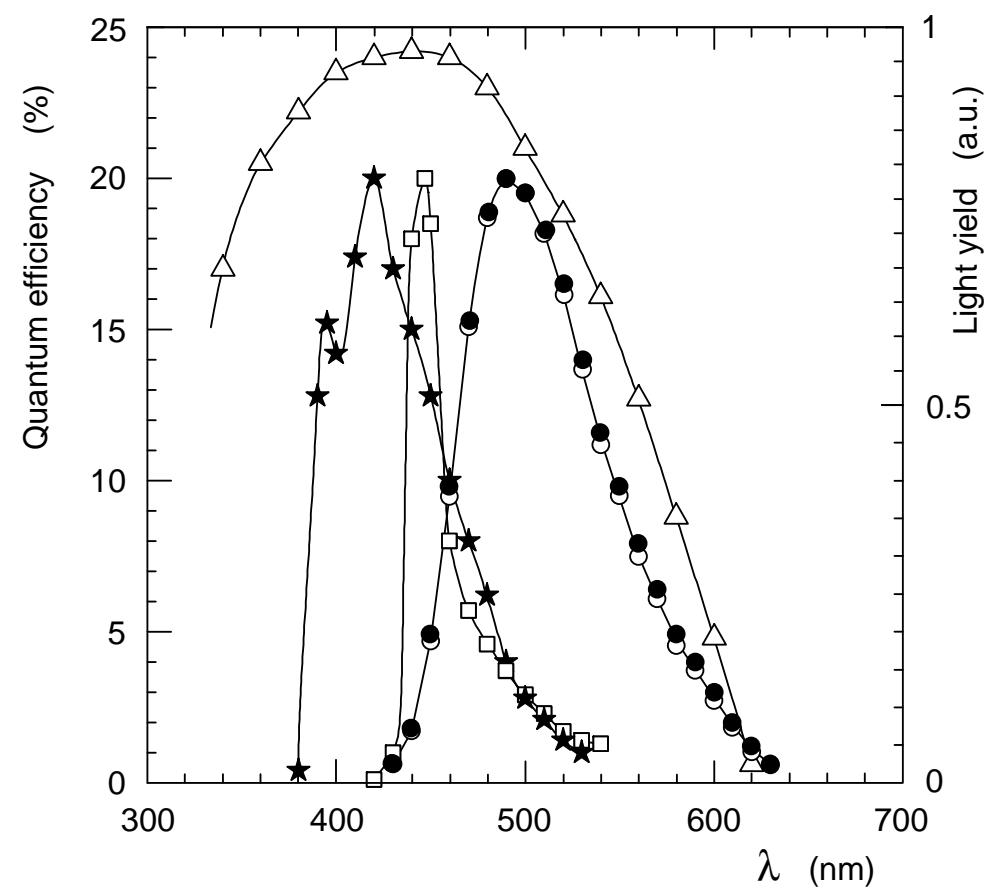

Figure 2: Quantum efficiency of our photomultiplier ( $\triangle$ and left scale) and emission spectra (right scale) of polystyrene scintillator $(\star)$, anthracene $(\square), 1 \mathrm{MN}+\mathrm{R} 6$ in vacuum $(\circ)$ and in air $(\bullet)$, when excited with a $365 \mathrm{~nm}$ wavelength.

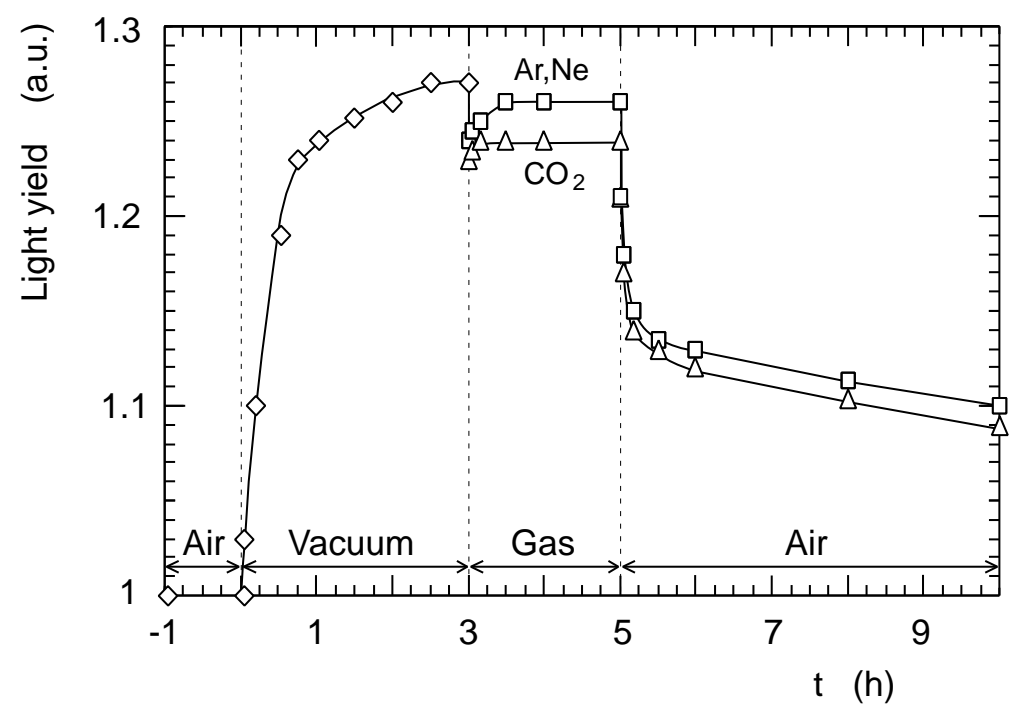

Figure 3: Time behaviour of the light yield of $1 \mathrm{MN}+\mathrm{R} 6$ scintillator at $20^{\circ} \mathrm{C}$, in air at normal pressure $(t<0 \mathrm{~h})$, when degassed in vacuum $(0<t<3 \mathrm{~h})$, when placed in a gas atmosphere $(3<t<5 \mathrm{~h})$, and when contact with air is restored $(t>5 \mathrm{~h})$. The curves refer to Ar and Ne gas (upper curve) and to $\mathrm{CO}_{2}$ (lower curve). The light yield in air was set to 1 . Similar curves are obtained for nitrogen and freon. 


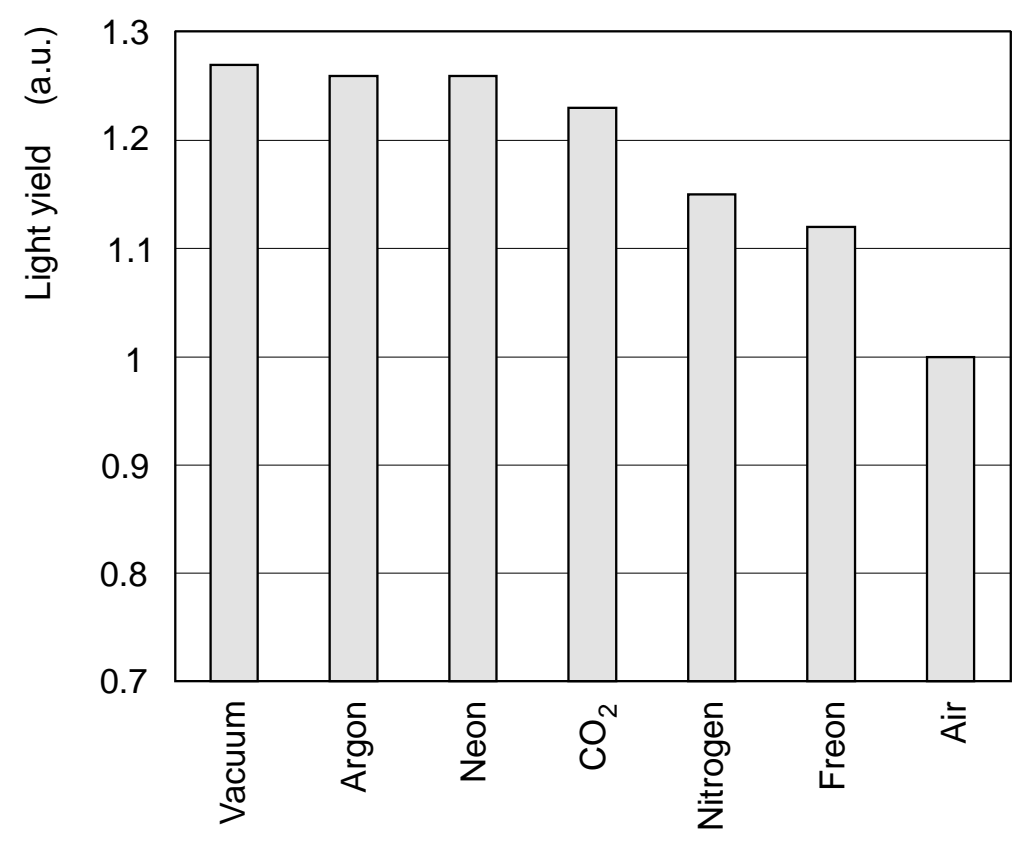

Figure 4: Relative light yield of $1 \mathrm{MN}+\mathrm{R} 6$ scintillator at $20^{\circ} \mathrm{C}$, when placed in an atmosphere of different gases. The light yield values refer to the plateau, in the time interval $4<t<5 \mathrm{~h}$ of Fig. 3. The light yield in air was set to 1 .

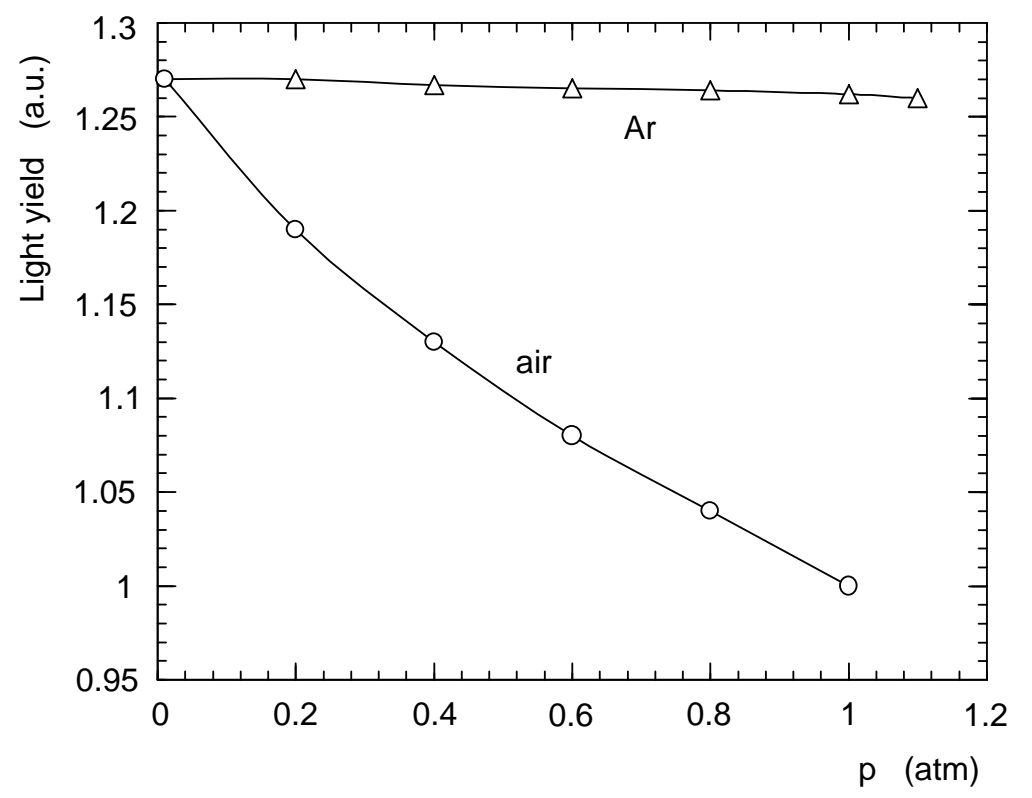

Figure 5: Light yield of $1 \mathrm{MN}+\mathrm{R} 6$ scintillator in an atmosphere of Ar (upper curve) or air (lower curve) as a function of the pressure of these gases. 


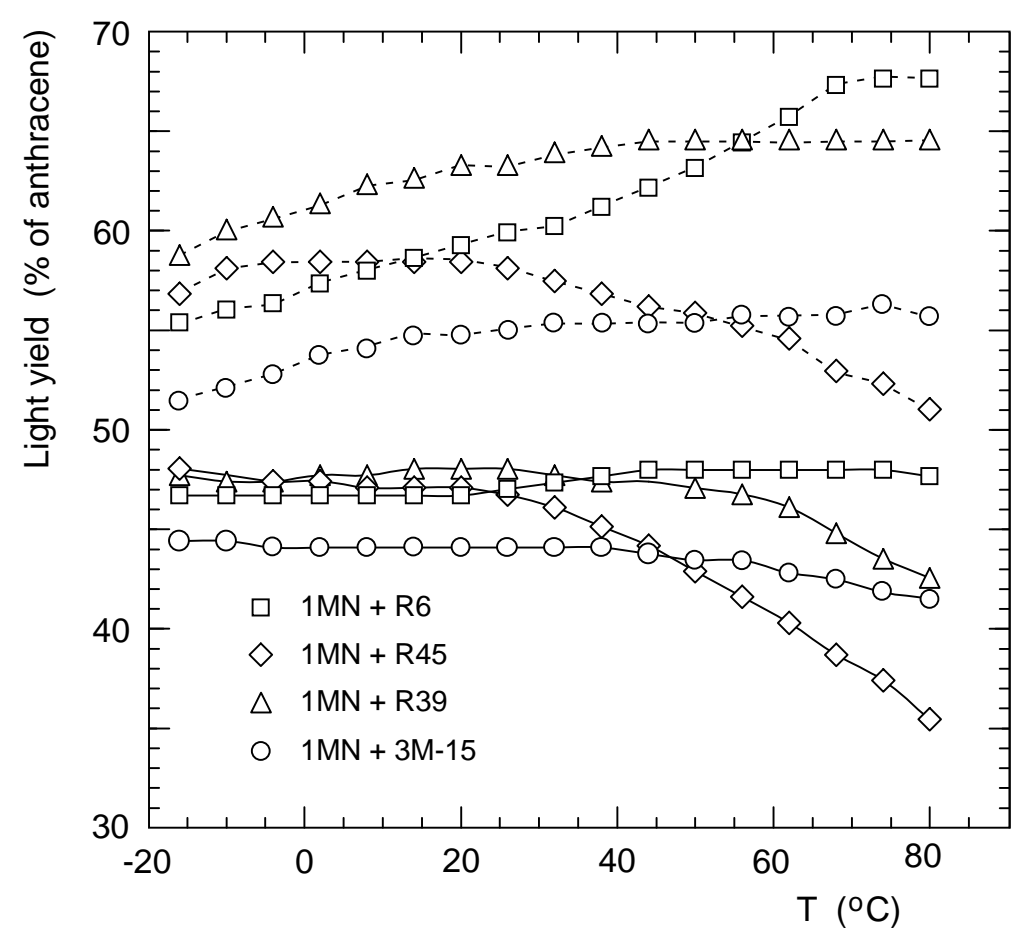

Figure 6: Light yield as a function of temperature of scintillators based on $1 \mathrm{MN}$, in air (solid lines) and in vacuum (dashed lines).

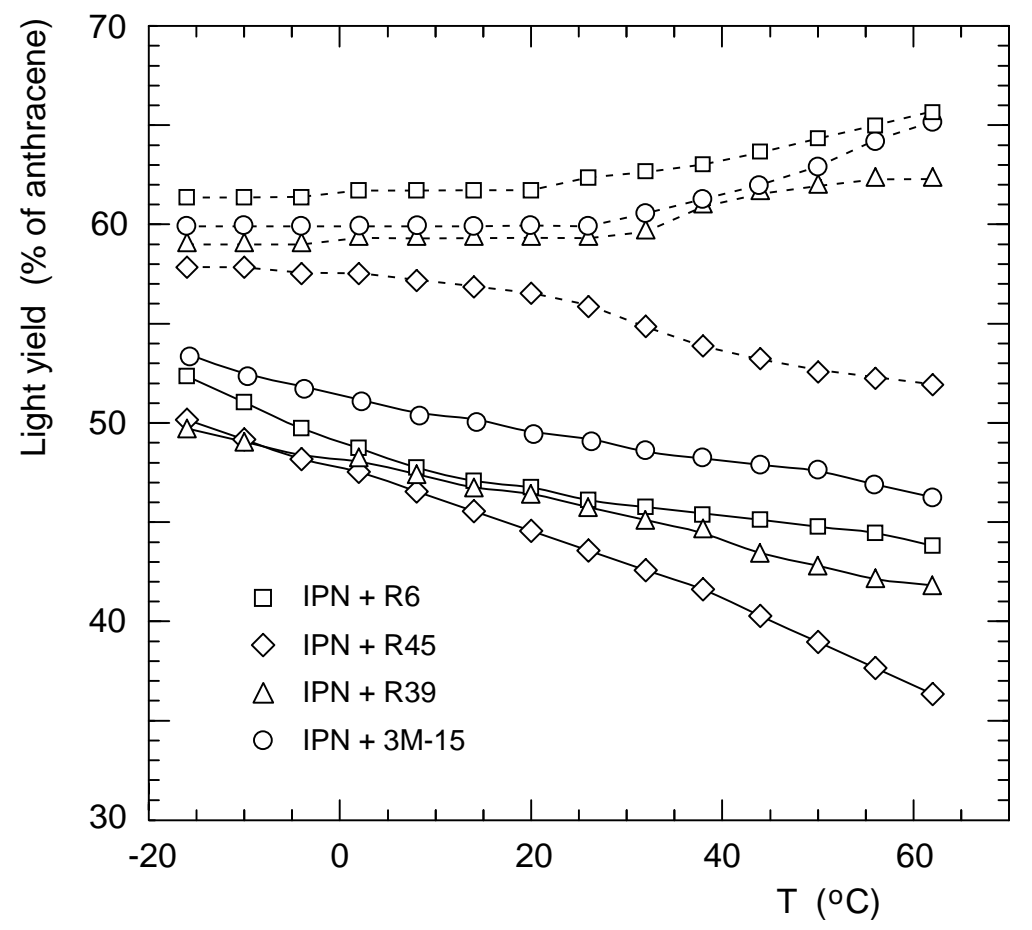

Figure 7: Light yield as a function of temperature of scintillators based on IPN, in air (solid lines) and in vacuum (dashed lines). 


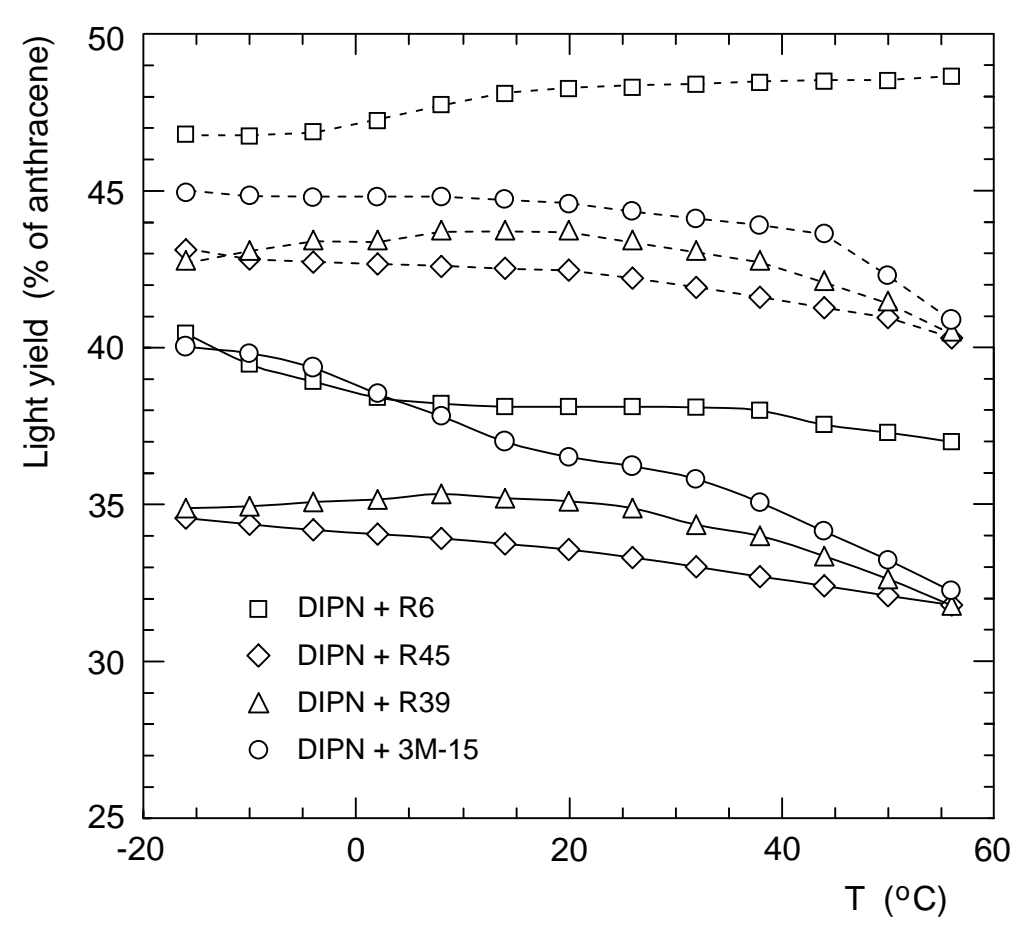

Figure 8: Light yield as a function of temperature of scintillators based on DIPN, in air (solid lines) and in vacuum (dashed lines).

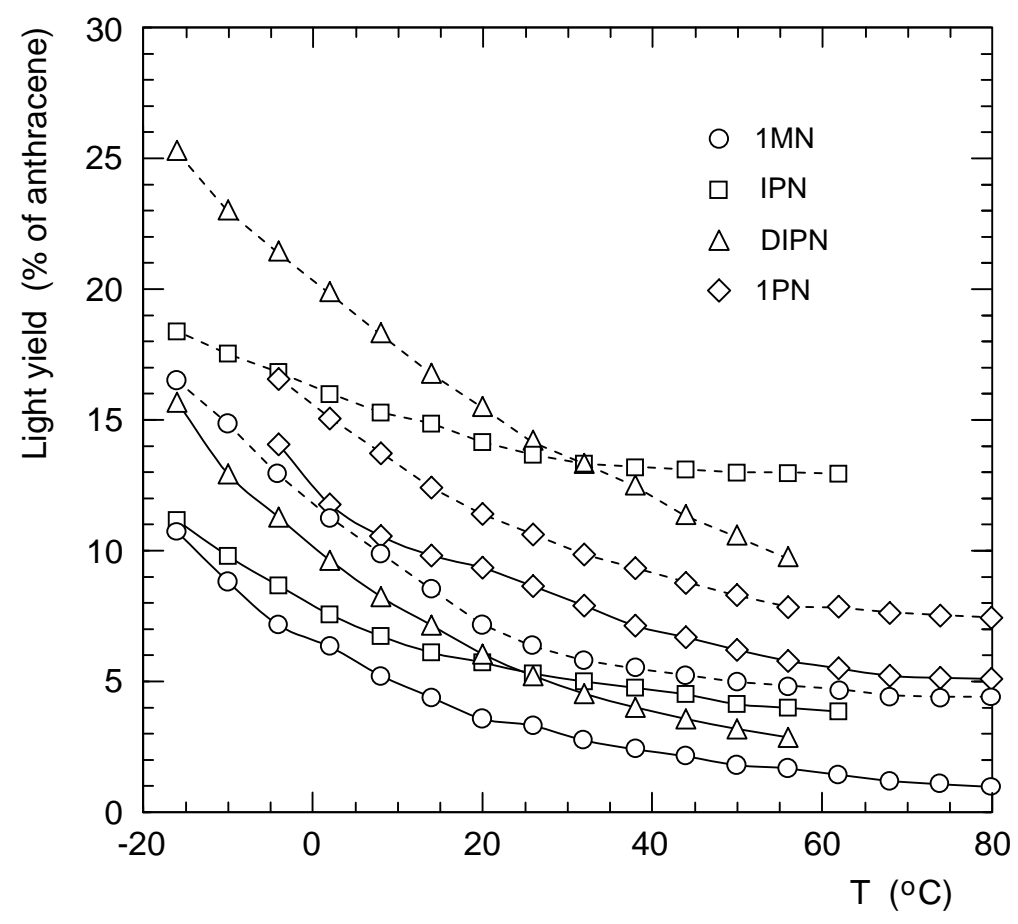

Figure 9: Light yield of pure solvents as a function of temperature, in air (solid lines) and in vacuum (dashed lines). 